\title{
The Quality Ratio of RAC Drawings and Planar Drawings of Planar Graphs
}

\author{
Marc van Kreveld \\ Department of Information and Computing Sciences, \\ Utrecht University, The Netherlands \\ marc@cs.uu.nl
}

\begin{abstract}
We study how much better a right-angled crossing (RAC) drawing of a planar graph can be than any planar drawing of the same planar graph. We analyze the area requirement, the edge-length ratio, and the angular resolution. For the first two measures, a RAC drawing can be arbitrarily much better, whereas for the third measure a RAC drawing can be 2.75 times as good.
\end{abstract}

Keywords: Right-angled crossing drawing, planar graphs, quality.

\section{Introduction}

Right-angled crossing drawings of graphs were introduced recently, motivated by the fact that good drawings may have crossings, as long as the crossing edges have a large crossing angle. In a right-angled crossing drawing (RAC drawing), every two edges that cross must do so at a right angle. It was shown that RAC drawings of graphs with $n$ vertices can have up to $4 n-10$ edges, and this bound is tight in the worst case [4].

Although RAC drawings can be drawings of non-planar graphs, one could also use a RAC drawing of planar graph in order to get a better angular resolution (for instance). For example, the $K_{4}$ has a RAC drawing whose smallest angle is $\pi / 4$ (the square with two diagonals), while any planar drawing has an angle of at most $\pi / 6$ (the optimum is realized by the equilateral triangle with the center point). Hence, the angular resolution of a RAC drawing can be 1.5 times as good as the angular resolution of any planar drawing of the same planar graph.

Let $\Phi$ be a quality measure of a drawing like area requirement, edge-length ratio, or angular resolution. In this paper we study how much better a RAC drawing of a planar graph can be than any planar drawing of the same graph. In particular, we study the quality ratio

$$
Q R(\Phi)=\sup _{G \text { planar }} \frac{\Phi_{\mathrm{RAC}}(G)}{\Phi_{\text {planar }}(G)},
$$

where $\Phi_{\mathrm{RAC}}(G)$ is the quality of the RAC drawing that is optimal for graph $G$ and measure $\Phi$, and $\Phi_{\text {planar }}(G)$ is the quality of the planar drawing that is optimal for graph $G$ and measure $\Phi$. While research on RAC drawings has 
considered both the case of straight-line drawings and drawings with bends in the edges [14], we consider only straight-line drawings.

Firstly, we consider the area requirement (Section 2). We show that for any $n$, there is a planar graph with $n$ vertices that admits a RAC drawing of area $O(n)$, but any planar drawing requires area $\Omega\left(n^{2}\right)$. This implies that a RAC drawing can be arbitrarily much better than a planar drawing of the same planar graph. If the area requirement quality $\Phi^{A}(G)$ is defined as the reciprocal of the minimum area needed in a drawing of $G$, then $Q R\left(\Phi^{A}\right)=\infty$.

Secondly, we consider the edge-length ratio (Section 3 ). We give a class of graphs that has constant edge-length ratio for RAC drawings but an unbounded edge-length ratio for all planar drawings when $n \rightarrow \infty$. We define the edge-length ratio quality $\Phi^{E}(G)$ as the longest possible length of the shortest edge in any drawing of $G$, assuming the longest edge in that drawing has length 1 . Then we will show that $Q R\left(\Phi^{E}\right)=\infty$.

Thirdly, we consider the angular resolution (Section 4). Let $\Phi^{\alpha}(G)$ be the largest possible smallest angle in a drawing of $G$. It follows from the results of Malitz and Papakostas [6] that for constant-degree planar graphs, the ratio of angular resolution is constant, because a planar drawing with all angles at least $\Omega\left(1 / 7^{d}\right)$ exists (where $d$ is the maximum degree; see also [5]), while no drawing - planar or not - can do better than $2 \pi / d$. We give a planar graph of degree 8 that shows that $Q R\left(\Phi^{\alpha}\right) \geq 2.75$. Whether $Q R\left(\Phi^{\alpha}\right)$ is bounded from above by a constant for planar graphs of non-constant degree is open.

\section{Ratio of Area Requirement}

Consider the class of graphs shown in Figure 1 (left). The $n$ vertices can be placed on a $2 \times n / 2$ grid for a RAC drawing, and hence the area requirement is linear in $n$. For any planar drawing, we show that it contains a linear number of nested triangles, and hence the area requirement of any planar drawing is quadratic 3 .

Consider the middle $K_{4}$ of the sequence, abcd. For a drawing to be planar, one of the vertices must be inside the triangle formed by the other three. Since the situation is symmetric, we can assume that $c$ is inside $\triangle a b d$ in a planar drawing. Since the vertices $e, f$ are adjacent to both $c$ and $d$, they must be inside $\triangle a b d$, and so must the whole further part of the graph beyond $e$ and $f$
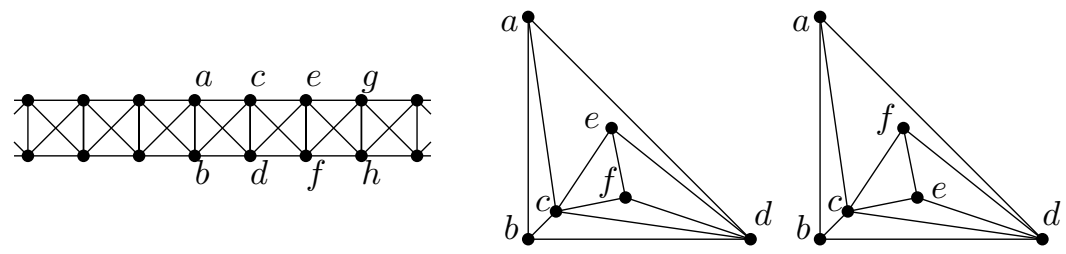

Fig. 1. Area requirement of a RAC drawing and a planar drawing 
including $g$ and $h$. Since the subgraph $c, d, e, f$ must be planar, we either have $f$ inside $\triangle c d e$ or $e$ inside $\triangle c d f$, see Figure 1 right. We repeat the argument once more to conclude that $h$ is inside $\triangle e f g$ or $g$ is inside $\triangle e f h$. In the first case, we have $\triangle a b d$ enclosing $\triangle e f g$, and the remainder of the graph (right part) is inside $\triangle e f g$. The second case is analogous. We conclude that in all cases, any planar drawing has $\Omega(n)$ nested triangles, and hence it has area requirement $\Omega\left(n^{2}\right)$. We conclude that the ratio of area-requirement quality is unbounded.

\section{Ratio of Edge-Length Ratio}

In this section we show that for a sufficiently large constant $R$, a graph exists such that a RAC drawing has edge-length ratio at most 3 and a planar drawing has edge-length ratio at least $R$. The graph consists of nested quadrilaterals, shown as squares in the left part of Figure 2. Nested squares come in pairs; note that the edges between the outer two squares are the same as the edges between the inner two squares. We can extend this construction to $\lfloor n / 10\rfloor$ pairs of squares in a graph with $n$ vertices. The graph has a unique embedding except for the choice of the outer face, and by a standard duplication of the construction we can ensure that any embedding has $\Omega(n)$ pairs of nested quadrilaterals. We will show that the inner quadrilateral of a pair is sufficiently smaller in some sense (area and/or diameter) than the outer quadrilateral of that pair. Since we have $\Omega(n)$ pairs of nested quadrilaterals and $n$ is not bounded by any constant, the innermost one can be made arbitrarily much smaller than the outermost one.
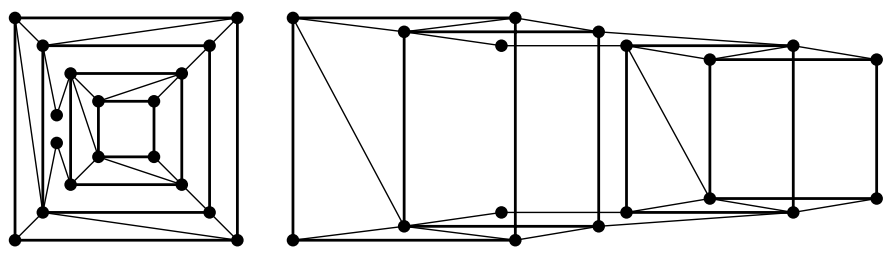

Fig. 2. Planar and RAC drawing of a graph that illustrates that the ratio of edge-length quality is unbounded

Figure 2 (right) shows that a RAC drawing exists where the edge-length ratio is less than 3 . The quadrilaterals can be drawn as squares that are not nested, and the drawing can be extended to any number of squares. If the outermost square has edge length 1 , then for any $\epsilon>0$, we can make the innermost square have edge length $1-\epsilon$, and the longest and shortest edges have lengths approximately $\frac{1}{2} \sqrt{5}$ and $\frac{1}{2}$.

Summarizing, we can prove unbounded ratio of edge-length quality for RAC drawings and planar drawings of the same graph if we can show that in any planar drawing of the graph shown in Figure 2, an edge-length ratio bounded by $R$ implies that the inner quadrilateral of a pair of nested quadrilaterals is smaller by some significant amount than the outer quadrilateral. 

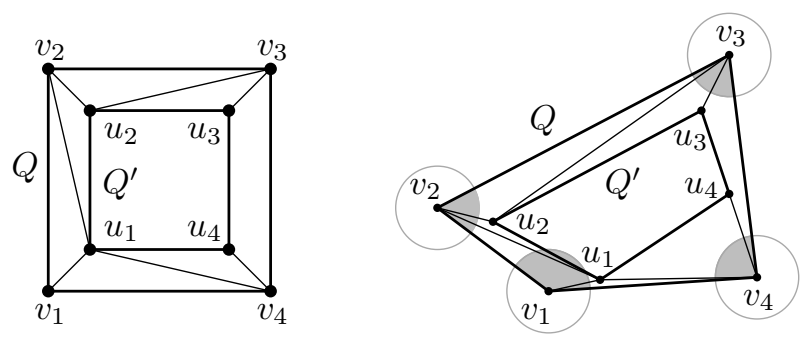

Fig. 3. The graph $G$ and its edges (left). The 1-neighborhood regions of the four vertices of $Q$.

Lemma 1. Let $G$ be a planar graph as in Figure 3, using the shown embedding. Then for any embedding-preserving straight-line drawing of $G$ and some large enough constant $R$, if the edge length ratio of $G$ is at most $R$, then the diameter ratio of the quadrilaterals $Q=v_{1} v_{2} v_{3} v_{4}$ and $Q^{\prime}=u_{1} u_{2} u_{3} u_{4}$ is greater than $1+\Omega\left(1 / R^{2}\right)$ or the area ratio of $Q$ and $Q^{\prime}$ is greater than $1+\Omega\left(1 / R^{4}\right)$.

Proof. Let $R$ be a sufficiently large constant. Consider any embedding-preserving straight-line drawing of $G$ with shortest edge length 1 and longest edge length $R$. Then the area of $Q$ is in $\left(0, R^{2}\right]$ and the diameter of $Q$ is in $(1,2 R)$. The area and diameter of $Q^{\prime}$ is always smaller than that of $Q$, but we will show that at least one of these measures is significantly smaller.

We define the 1-neighborhoods of $v_{1}, \ldots, v_{4}$ as the intersection of the unit disks centered at $v_{1}, \ldots, v_{4}$ with the interior of $Q$. By the edges of $G$ between $Q$ and $Q^{\prime}, u_{i}$ cannot be in the 1-neighborhood of $v_{i}$ for $i=1, \ldots, 4$, and $u_{1}$ cannot be in the 1-neighborhood of $v_{2}$ or $v_{4}$, and $u_{2}$ cannot be in the 1-neighborhood of $v_{3}$.

If none of $u_{1}, \ldots, u_{4}$ is in the 1-neighborhood of any of $v_{1}, \ldots, v_{4}$, then the diameter decreases at least by some constant amount, depending on $R$. Since $R$ is a constant, the diameter of $Q^{\prime}$ is smaller by a constant fraction $<1$ (some calculation shows that it is at most $1-\frac{c}{R^{2}}$ times the diameter of $Q$, for a constant $c>0)$. So it remains to analyze the cases where at least some $u_{1}, \ldots, u_{4}$ are in the 1-neighborhood of some of $v_{1}, \ldots, v_{4}$, but with the restrictions on which $u_{i}$ cannot be close to which $v_{j}$.

Consider the six triangles of $G$ in between $Q$ and $Q^{\prime}$ (we ignore the quadrilateral that is also in between). If any of these triangles has at least a constant area (which may depend on $R$ ), then the area of $Q^{\prime}$ is a constant fraction $<1$ less than the area of $Q$. In particular, this implies that $u_{1}$ must be very close to the line through $v_{1}$ and $v_{2}$ (due to the area of $\triangle u_{1} v_{1} v_{2}$ and the shortest edge length of 1) and very close to the line through $v_{1}$ and $v_{4}$ (due to the area of $\triangle u_{1} v_{1} v_{4}$ and the shortest edge length of 1$)$. In particular, a distance of at least $1 / R^{2}$ implies that the area of a triangle is at least $1 /\left(2 R^{2}\right)$, which makes the lemma true because the area of $Q$ is at most $R^{2}$. So we continue with the case where the distance from $u_{1}$ to these lines is less than $1 / R^{2}$. Since $u_{1}$ is close to 
both lines but at least distance 1 from $v_{1}$, we see that the angle at $v_{1}$ in $Q$ is either very close to 0 , or very close to 180 , or very close to 360 degrees (within $c / R^{2}$ for some constant $c>0$ ).

By analyzing these three cases for the angle at $v_{1}$ and many subcases, we can always conclude that either the area of $Q^{\prime}$ or the diameter is significantly smaller than that of $Q$ (or else we get a contradiction with the planarity of $G$ ). The rather tedious full proof is in the full paper.

By applying the lemma above to a graph with $k$ pairs of nested quadrilaterals, the outermost quadrilateral has a diameter that is at least $\left(1+\Omega\left(1 / R^{2}\right)\right)^{k / 2}$ times as large as the innermost one, or the outermost quadrilateral has an area at least $\left(1+\Omega\left(1 / R^{4}\right)\right)^{k / 2}$ times as large as the innermost one. By choosing $k$ (and therefore $n$ ) large enough, we can achieve a diameter ratio or area ratio larger than any constant, because $R$ is assumed to be constant. Since an upper bound on the diameter or the area (independent of each other) bound the maximum edge length that is possible, we derive a contradiction that follows from the assumption that $R$ is constant. We conclude that the edge-length ratio for any planar drawing of the graph is unbounded, and the quality ratio of edge length is unbounded as well.

\section{Ratio of Angular Resolution}

That the angular resolution of a RAC drawing can be better than of a planar drawing can easily be seen from the $K_{4}$. A RAC drawing can be a square with the two diagonals, giving a smallest angle of $45^{\circ}$, while a planar drawing with optimal angular resolution is an equilateral triangle that has its fourth vertex in the center, giving a smallest angle of $30^{\circ}$. The quality ratio is 1.5 . By extending the example we obtain another planar graph that has a RAC drawing with smallest angle $45^{\circ}$, see Figure 4, left. Any planar drawing must have one of $a, b, c, d$ inside the triangle formed by the other three. Since the graph is symmetric, we can assume that $d$ is inside. But then the biconnected component shown below $b, d$ and the biconnected component right of $c, d$ must be inside $\triangle a b c$ as well. This implies that $\triangle a b c$ has its angle at $a$ partitioned into two angles, its angle
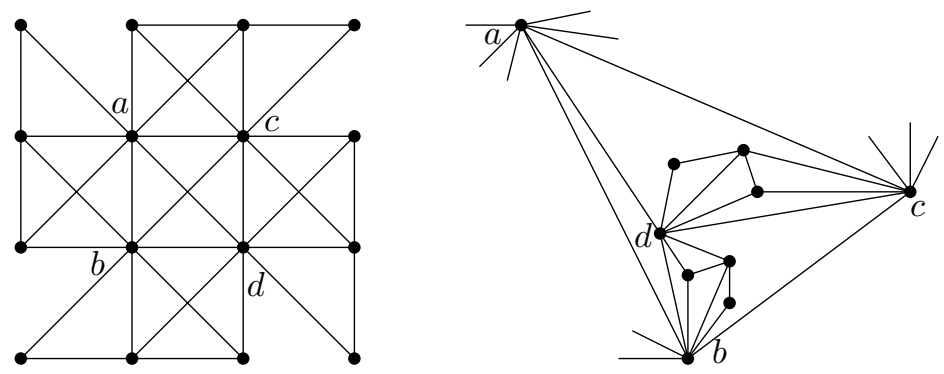

Fig. 4. A planar graph showing that the ratio of angular resolution is at least $11 / 4$ 
at $b$ partitioned in five angles, and its angle at $c$ partitioned into four angles by edges from these vertices. In total, the angle of $180^{\circ}$ for $\triangle a b c$ is split into eleven parts, so there will be some angle of at most $\frac{180}{11}^{\circ}$ in any planar drawing of the graph. Hence, the ratio of angular resolution is at least $11 / 4=2.75$.

\section{Conclusions and Further Research}

This paper compared quality measures of RAC drawings and planar drawings by defining a ratio, using the best possible RAC drawing and planar drawing of the same graph, maximized over all planar graphs. We showed that the quality ratio for area requirement quality is unbounded, and so is the quality ratio of edge-length. For angular resolution we gave an example that shows that the quality ratio is at least 2.75 . It is unknown - and the main open problem arising from this paper - whether this quality ratio is bounded by a constant or not. If it is bounded by a constant, it may still be possible to improve upon the given bound of 2.75 .

Several other aesthetic criteria can also be analyzed. A list is given in [2]. Our construction that shows an unbounded quality ratio of edge length also shows that the quality ratio of total edge length is unbounded, assuming the shortest edge has unit length. For the criterion of symmetry, a RAC drawing of a $K_{4}$ using a square is in a sense "more symmetric" than any planar drawing of a $K_{4}$. In a $K_{4}$, all vertices are "the same", which is also true in the RAC drawing but not in any planar drawing.

This paper considered only straight-line drawings of planar graphs. One can extend the research to drawings with bends and analyze the ratio of RAC drawings and planar drawings in this case as well. This is left for future research.

\section{References}

1. Angelini, P., Cittadini, L., Battista, G.D., Didimo, W., Frati, F., Kaufmann, M., Symvonis, A.: On the perspectives opened by right angle crossing drawings. In: Eppstein, D., Gansner, E.R. (eds.) GD 2009. LNCS, vol. 5849, pp. 21-32. Springer, Heidelberg (2010)

2. Battista, G.D., Eades, P., Tamassia, R., Tollis, I.: Graph Drawing: Algorithms for the Visualization of Graphs. Prentice-Hall, Englewood Cliffs (1999)

3. de Fraysseix, H., Pach, J., Pollack, R.: How to draw a planar graph on a grid. Combinatorica 10, 41-51 (1990)

4. Didimo, W., Eades, P., Liotta, G.: Drawing graphs with right angle crossings. In: Dehne, F., Gavrilova, M., Sack, J.-R., Tóth, C.D. (eds.) WADS 2009. LNCS, vol. 5664, pp. 206-217. Springer, Heidelberg (2009)

5. Garg, A., Tamassia, R.: Planar drawings and angular resolution: Algorithms and bounds (extended abstract). In: van Leeuwen, J. (ed.) ESA 1994. LNCS, vol. 855, pp. 12-23. Springer, Heidelberg (1994)

6. Malitz, S., Papakostas, A.: On the angular resolution of planar graphs. SIAM J. Discrete Math. 7(2), 172-183 (1994) 